\title{
Fine gradings and gradings by root systems on simple Lie algebras
}

\author{
Alberto Elduque
}

\begin{abstract}
Given a fine abelian group grading $\Gamma: \mathcal{L}=\bigoplus_{g \in G} \mathcal{L}_{g}$ on a finite dimensional simple Lie algebra over an algebraically closed field of characteristic zero, with $G$ being the universal grading group, it is shown that the induced grading by the free group $G / \operatorname{tor}(G)$ on $\mathcal{L}$ is a grading by a (not necessarily reduced) root system.

Some consequences for the classification of fine gradings on the exceptional simple Lie algebras are deduced.
\end{abstract}

\section{Introduction}

Gradings by abelian groups on simple Lie algebras appear in many situations. A systematic study of these gradings was started in [28]. For the classical simple Lie algebras over an algebraically closed field of characteristic 0 , the fine gradings were classified in [18]. For the exceptional simple algebras they were classified in [12] and [6] for $G_{2}$, in [13] for $F_{4}$, and in [14] for $E_{6}$. See also [20].

On the other hand, gradings by root systems were introduced by Berman and Moody in [9], who used them as tools to study some classes of infinite-dimensional Lie algebras.

The goal of this paper is to relate both types of gradings. It will be shown that any fine grading with infinite universal grading group on a simple finite-dimensional Lie algebra over an algebraically closed field of characteristic 0 induces a grading by a (possibly nonreduced) root system. Some consequences for the classification of fine gradings in the exceptional cases will be derived too.

The next two sections review gradings by abelian groups and gradings by root systems respectively. The main result connecting fine gradings and gradings by root systems is proved in the following two sections. This result shows that any fine grading is determined by a grading by a root system and a special grading on

Mathematics Subject Classification (2010): Primary 17B70; Secondary 17B22, 17B25, 17B60.

Keywords: Fine grading, simple Lie algebra, grading by root systems, exceptional, coordinate algebra. 
the coordinate algebra of the root grading. This grading on the coordinate algebra is studied in Section 6. The last section is devoted to deducing consequences for the classification of the fine gradings on the simple exceptional simple Lie algebras.

\section{Gradings}

Let $\mathcal{A}$ be an algebra (not necessarily associative) over a field $\mathbb{F}$ and let $G$ be an abelian group (written additively).

Definition 2.1. A $G$-grading on $\mathcal{A}$ is a vector space decomposition

$$
\Gamma: \mathcal{A}=\bigoplus_{g \in G} \mathcal{A}_{g}
$$

such that

$$
\mathcal{A}_{g} \mathcal{A}_{h} \subset \mathcal{A}_{g+h} \text { for all } g, h \in G \text {. }
$$

If such a decomposition is fixed, we refer to $\mathcal{A}$ as a $G$-graded algebra. The nonzero elements $a \in \mathcal{A}_{g}$ are said to be homogeneous of degree $g$; we write $\operatorname{deg} a=g$. The support of $\Gamma$ is the set Supp $\Gamma:=\left\{g \in G \mid \mathcal{A}_{g} \neq 0\right\}$.

Let

$$
\Gamma: \mathcal{A}=\bigoplus_{g \in G} \mathcal{A}_{g} \quad \text { and } \quad \Gamma^{\prime}: \mathcal{B}=\bigoplus_{h \in H} \mathcal{B}_{h}
$$

be two gradings on algebras, with supports $S$ and $T$, respectively.

Definition 2.2. We say that $\Gamma$ and $\Gamma^{\prime}$ are equivalent if there exists an isomorphism of algebras $\psi: \mathcal{A} \rightarrow \mathcal{B}$ and a bijection $\alpha: S \rightarrow T$ such that $\psi\left(\mathcal{A}_{s}\right)=\mathcal{B}_{\alpha(s)}$ for all $s \in S$. Any such $\psi$ is called an equivalence of $\Gamma$ and $\Gamma^{\prime}$ (or of $\mathcal{A}$ and $\mathcal{B}$ if the gradings are clear from the context).

Given a group grading $\Gamma$ on an algebra $\mathcal{A}$, there are many groups $G$ such that $\Gamma$, regarded as a direct sum of subspaces such that the product of any two lies in a third, can be realized as a $G$-grading, but among such groups, there is a distinguished one [28].

Definition 2.3. Suppose that $\Gamma$ admits a realization as a $U$-grading for some abelian group $U$. We say that $U$ is a universal group of $\Gamma$ if, for any other realization of $\Gamma$ as a $G$-grading, there exists a unique homomorphism $U \rightarrow G$ that restricts to the identity on Supp $\Gamma$.

One shows that the universal group, which we denote by $U(\Gamma)$, exists and depends, up to isomorphism, only on the equivalence class of $\Gamma$. Indeed, $U(\Gamma)$ is generated by $S=\operatorname{Supp} \Gamma$ with defining relations $s_{1}+s_{2}=s_{3}$ whenever $0 \neq$ $\mathcal{A}_{s_{1}} \mathcal{A}_{s_{2}} \subset \mathcal{A}_{s_{3}}\left(s_{i} \in S\right)$. 
Given a $G$-grading $\Gamma: \mathcal{A}=\bigoplus_{g \in G} \mathcal{A}_{g}$ and a group homomorphism $\alpha: G \rightarrow H$, we obtain the induced $H$-grading

$$
{ }^{\alpha} \Gamma: \mathcal{A}=\bigoplus_{h \in H} \mathcal{A}_{h}^{\prime}
$$

by setting

$$
\mathcal{A}_{h}^{\prime}=\bigoplus_{g \in \alpha^{-1}(h)} \mathcal{A}_{g} .
$$

Definition 2.4. Given gradings $\Gamma: \mathcal{A}=\bigoplus_{g \in G} \mathcal{A}_{g}$ and $\Gamma^{\prime}: \mathcal{A}=\bigoplus_{h \in H} \mathcal{A}_{h}^{\prime}$, we say that $\Gamma^{\prime}$ is a coarsening of $\Gamma$, or that $\Gamma$ is a refinement of $\Gamma^{\prime}$, if for any $g \in G$ there exists $h \in H$ such that $\mathcal{A}_{g} \subset \mathcal{A}_{h}^{\prime}$. The coarsening (or refinement) is said to be proper if the inclusion is proper for some $g \in \operatorname{Supp} \Gamma$. (In particular, ${ }^{\alpha} \Gamma$ is a coarsening of $\Gamma$, which is not necessarily proper.) A grading $\Gamma$ is said to be fine if it does not admit a proper refinement.

Any $G$-grading on a finite-dimensional algebra $\mathcal{A}$ is induced from some fine grading $\Gamma$ by a homomorphism $\alpha: U(\Gamma) \rightarrow G$.

Over algebraically closed fields of characteristic zero, the classification of fine gradings on $\mathcal{A}$ up to equivalence is the same as the classification of maximal diagonalizable subgroups (i.e., maximal quasitori) of $\operatorname{Aut}(\mathcal{A})$ up to conjugation (see e.g. [28]). More precisely, given a grading $\Gamma$ on the algebra $\mathcal{A}$ with universal group $G$, let $\hat{G}$ be its group of characters (homomorphisms $G \rightarrow \mathbb{F}^{\times}$). Any $\chi \in \hat{G}$ acts as an automorphism of $\mathcal{A}$ by means of $\chi \cdot x=\chi(g) x$ for any $g \in G$ and $x \in \mathcal{A}_{g}$. This allows us to identify $\hat{G}$ with a quasitorus (the direct product of a torus and a finite subgroup) of the algebraic group $\operatorname{Aut}(\mathcal{A})$. Conversely, given a quasitorus $Q$ of $\operatorname{Aut}(\mathcal{A}), Q=\hat{G}$ for $G$ the group of homomorphisms (as algebraic groups) $Q \rightarrow \mathbb{F}^{\times}$. Then $Q$ induces a $G$-grading of $\mathcal{A}$, where $\mathcal{A}_{g}=\{x \in \mathcal{A}: \chi(x)=g(\chi) x\}$ for any $g \in G$. In this way [28], the fine gradings on $\mathcal{A}$, up to equivalence, correspond to the conjugacy classes in $\operatorname{Aut}(\mathcal{A})$ of the maximal quasitori (or maximal abelian diagonalizable subgroups) of $\operatorname{Aut}(\mathcal{A})$.

Fine gradings on simple Lie algebras belonging to the series $A, B, C$, and $D$ (including $D_{4}$ ) were classified in [18]. The classifications of the fine gradings on simple Lie algebras of exceptional type were obtained, for type $G_{2}$ in [12], [6], for type $F_{4}$ in [13] (see also [19]), and for type $E_{6}$ in [14].

Definition 2.5. Let $\Gamma: \mathcal{A}=\bigoplus_{g \in G} \mathcal{A}_{g}$ be a grading on the algebra $\mathcal{A}$.

- A subspace $\mathcal{B}$ of $\mathcal{A}$ is said to be graded if $\mathcal{B}=\bigoplus_{g \in G}\left(\mathcal{B} \cap \mathcal{A}_{g}\right)$. (Equivalently, $\mathcal{B}$ is graded by $G$ with $\mathcal{B}_{g}=\mathcal{B} \cap \mathcal{A}_{g}$ for any $g \in G$.)

- The type of $\Gamma$ is the $r$-tuple $\left(n_{1}, \ldots, n_{r}\right)$, where $r=\max \left\{\operatorname{dim} \mathcal{A}_{g}: g \in G\right\}$ and $n_{i}$ is the number of homogeneous components of dimension $i$, for $i=1, \ldots, r$. Hence $\operatorname{dim} \mathcal{A}=\sum_{i=1}^{r} i n_{i}$.

From now on, the ground field $\mathbb{F}$ will be assumed to be algebraically closed of characteristic zero. 


\section{Gradings by root systems}

Berman and Moody [9] started the study of Lie algebras graded by root systems $\Phi$. (See [3] and the references therein.)

Definition 3.1. A Lie algebra $\mathcal{L}$ over $\mathbb{F}$ is graded by the reduced root system $\Phi$, or $\Phi$-graded, if:

(i) $\mathcal{L}$ contains as a subalgebra a finite-dimensional simple Lie algebra $\mathfrak{g}=\mathfrak{h} \oplus$ $\left(\bigoplus_{\alpha \in \Phi} \mathfrak{g}_{\alpha}\right)$ whose root system is $\Phi$ relative to a Cartan subalgebra $\mathfrak{h}=\mathfrak{g}_{0}$;

(ii) $\mathcal{L}=\bigoplus_{\alpha \in \Phi \cup\{0\}} \mathcal{L}(\alpha)$, where $\mathcal{L}(\alpha)=\{X \in \mathcal{L}:[H, X]=\alpha(H) X$ for all $H \in \mathfrak{h}\} ;$ and

(iii) $\mathcal{L}(0)=\sum_{\alpha \in \Phi}[\mathcal{L}(\alpha), \mathcal{L}(-\alpha)]$.

The subalgebra $\mathfrak{g}$ is said to be a grading subalgebra of $\mathcal{L}$.

Berman and Moody [9] studied the simply laced cases $A_{r}(r \geq 2), D_{r}$ and $E_{r}$, and Benkart and Zelmanov [8] considered the remaining cases.

Under the adjoint action of $\mathfrak{g}$, a $\Phi$-graded Lie algebra $\mathcal{L}$ decomposes as a sum of finite-dimensional irreducible $\mathfrak{g}$-modules whose highest weights are the highest long root, highest short root, or 0. By collecting isomorphic summands into "isotypic components", we may assume that there are $\mathbb{F}$-vector spaces $\mathcal{A}, \mathcal{B}$, and $\mathcal{D}$ such that

$$
\mathcal{L}=(\mathfrak{g} \otimes \mathcal{A}) \oplus(\mathcal{W} \otimes \mathcal{B}) \oplus \mathcal{D},
$$

where the grading subalgebra $\mathfrak{g}$ is identified with $\mathfrak{g} \otimes 1$ for a distinguished element $1 \in \mathcal{A} ; \mathcal{W}$ is 0 if $\mathfrak{g}$ is of type $A_{r}(r \geq 1), D_{r}(r \geq 4)$, or $E_{r}(r=6,7,8)$, while $\mathcal{W}$ is the irreducible $\mathfrak{g}$-module whose highest weight is the highest short root if $\mathfrak{g}$ is of type $B_{r}(r \geq 2), C_{r}(r \geq 3), F_{4}$, or $G_{2}$; and $\mathcal{D}$ is the centralizer of $\mathfrak{g} \simeq \mathfrak{g} \otimes 1$, and hence it is a subalgebra of $\mathcal{L}$.

The problem of classifying the $\Phi$-graded Lie algebras reduces to that of determining the possibilities for $\mathcal{A}, \mathcal{B}$, and $\mathcal{D}$, and of finding the multiplication. The bracket in $\mathcal{L}$ is invariant under the adjoint action of $\mathfrak{g}$ and this gives the sum $\mathfrak{a}=\mathcal{A} \oplus \mathcal{B}$ the structure of a unital algebra. Moreover, $\mathcal{D}$ acts as derivations on $\mathfrak{a}$, with $\mathcal{A}$ and $\mathcal{B}$ being invariant under this action. The type of the algebra $\mathfrak{a}$ depends on the root system $\Phi$. This algebra $\mathfrak{a}$ is called the coordinate algebra of $\mathcal{L}$.

For instance (see [8]), assume that $\Phi$ is the root system of type $G_{2}$. Then $\mathfrak{g}$ is the Lie algebra of type $G_{2}$, which can be identified with the Lie algebra of derivations of the Cayley (or octonion algebra) $\mathbb{O}$, and $\mathcal{W}$ can be identified with the subspace of trace zero octonions $\mathbb{O}_{0}$. The Cayley algebra is endowed with a nondegenerate quadratic form $n$ (the norm) such that any element $w$ satisfies $w^{2}-t(w) w+n(w) 1=0$, where $t(w)=n(w, 1):=n(w+1)-n(w)-1$.

Moreover, one has the following properties:

1. $\operatorname{Hom}_{\mathfrak{g}}(\mathfrak{g} \otimes \mathfrak{g}, \mathfrak{g})$ is spanned by the bracket.

2. $\operatorname{Hom}_{\mathfrak{g}}(\mathfrak{g} \otimes \mathfrak{g}, \mathbb{F})$ is spanned by the Killing form $\kappa$, which is a scalar multiple of the trace form relative to the representation provided by $\mathcal{W}$. 
3. $\operatorname{Hom}_{\mathfrak{g}}(\mathfrak{g} \otimes \mathcal{W}, \mathcal{W})$ is spanned by the action of $\mathfrak{g}$ on $\mathcal{W}(X \otimes W \mapsto X . W)$.

4. $\operatorname{Hom}_{\mathfrak{g}}(\mathcal{W} \otimes \mathcal{W}, \mathfrak{g})$ is spanned by the map $w_{1} \otimes w_{2} \mapsto D_{w_{1}, w_{2}}$, where $D_{w_{1}, w_{2}}(w)=$ $\left[\left[w_{1}, w_{2}\right], w\right]+3\left(\left(w_{1} w\right) w_{2}-w_{1}\left(w w_{2}\right)\right)$.

5. $\operatorname{Hom}_{\mathfrak{g}}(\mathcal{W} \otimes \mathcal{W}, \mathcal{W})$ is spanned by the bracket (inside $\left.\mathbb{O}\right) w_{1} \otimes w_{2} \mapsto\left[w_{1}, w_{2}\right]=$ $w_{1} w_{2}-w_{2} w_{1}$.

6. $\operatorname{Hom}_{\mathfrak{g}}(\mathcal{W} \otimes \mathcal{W}, \mathbb{F})$ is spanned by the trace form $w_{1} \otimes w_{2} \mapsto t\left(w_{1} w_{2}\right)$.

7. $\operatorname{Hom}_{\mathfrak{g}}(\mathfrak{g} \otimes \mathfrak{g}, \mathcal{W}), \operatorname{Hom}_{\mathfrak{g}}(\mathfrak{g} \otimes \mathcal{W}, \mathfrak{g})$, and $\operatorname{Hom}_{\mathfrak{g}}(\mathfrak{g} \otimes \mathcal{W}, \mathbb{F})$ are trivial.

Therefore, the bracket in $\mathcal{L}$ is given by:

$$
\begin{aligned}
{\left[D \otimes a, D^{\prime} \otimes a^{\prime}\right] } & =\left[D, D^{\prime}\right] \otimes a \cdot a^{\prime}+\kappa\left(D, D^{\prime}\right)\left\langle a \mid a^{\prime}\right\rangle \\
{[D \otimes a, w \otimes b] } & =D(w) \otimes a \cdot b \\
{[d, D \otimes a] } & =D \otimes d(a), \\
{[d, w \otimes b] } & =w \otimes d(b) \\
{\left[w \otimes b, w^{\prime} \otimes b^{\prime}\right] } & =D_{w, w^{\prime}} \otimes\left(b \mid b^{\prime}\right)+\left[w, w^{\prime}\right] \otimes b \circ b^{\prime}+2 t\left(w_{1} w_{2}\right)\left\langle b \mid b^{\prime}\right\rangle,
\end{aligned}
$$

for any $D, D^{\prime} \in \mathfrak{g} ; w, w^{\prime} \in \mathcal{W} ; a, a^{\prime} \in \mathcal{A} ; b, b^{\prime} \in \mathcal{B}$; and $d, d^{\prime} \in \mathcal{D}$; and for linear maps

- $\mathcal{A} \otimes \mathcal{A} \rightarrow \mathcal{A}: a \otimes a^{\prime} \mapsto a \cdot a^{\prime}$, which is symmetric;

- $\mathcal{A} \otimes \mathcal{A} \rightarrow \mathcal{D}: a \otimes a^{\prime} \mapsto\left\langle a \mid a^{\prime}\right\rangle$, which is skew-symmetric;

- $\mathcal{B} \otimes \mathcal{B} \rightarrow \mathcal{A}: b \otimes b^{\prime} \mapsto\left(b \mid b^{\prime}\right)$, which is symmetric;

- $\mathcal{B} \otimes \mathcal{B} \rightarrow \mathcal{B}: b \otimes b^{\prime} \mapsto b \circ b^{\prime}$, which is symmetric;

- $\mathcal{B} \otimes \mathcal{B} \rightarrow \mathcal{D}: b \otimes b^{\prime} \mapsto\left\langle b \mid b^{\prime}\right\rangle$, which is skew-symmetric;

- $\mathcal{A} \otimes \mathcal{B} \rightarrow \mathcal{B}: a \otimes b \mapsto a \cdot b$.

These linear maps satisfy the following properties:

1. $\mathcal{A}$ is a unital commutative algebra with the product $a \cdot a^{\prime}$.

2. $\mathfrak{a}=\mathcal{A} \oplus \mathcal{B}$ with the multiplication given by

$$
(a+b) \cdot\left(a^{\prime}+b^{\prime}\right)=\left(a \cdot a^{\prime}+\left(b \mid b^{\prime}\right)\right)+\left(a \cdot b^{\prime}+a^{\prime} \cdot b+b \circ b^{\prime}\right),
$$

for $a, a^{\prime} \in \mathcal{A}$ and $b, b^{\prime} \in \mathcal{B}$, is a Jordan algebra over $\mathcal{A}$ with normalized trace given by $\operatorname{trace}(a+b)=a$, which satisfies the Cayley-Hamilton equation of degree 3 .

3. The action of $\mathcal{D}$ on $\mathfrak{a}=\mathcal{A} \oplus \mathcal{B}$ is an action by derivations. Moreover, $\left\langle a \mid a^{\prime}\right\rangle(\mathfrak{a})=0=\left\langle b \mid b^{\prime}\right\rangle(\mathcal{A})$ and $\left\langle b^{\prime} \mid b^{\prime \prime}\right\rangle(b)=b^{\prime} \cdot\left(b^{\prime \prime} \cdot b\right)-b^{\prime \prime} \cdot\left(b^{\prime} \cdot b\right)$, for $a, a^{\prime} \in \mathcal{A}$ and $b, b^{\prime}, b^{\prime \prime} \in \mathcal{B}$.

4. $\mathcal{D}=\langle\mathcal{A} \mid \mathcal{A}\rangle+\langle\mathcal{B} \mid \mathcal{B}\rangle$. (This is implied by condition (iii) in Definition 3.1)

Therefore, in this case, the coordinate algebra $\mathfrak{a}$ is a Jordan algebra "of degree 3" over the unital commutative associative algebra $\mathcal{A}$ (see [8]).

Note that $\langle\mathcal{A} \mid \mathcal{A}\rangle$ is a central ideal of $\mathcal{L}$, so if $\mathcal{L}$ is simple, then $\langle\mathcal{A} \mid \mathcal{A}\rangle$ is trivial, and hence $\mathcal{D}=\langle\mathcal{B} \mid \mathcal{B}\rangle$. 
Gradings by nonreduced root systems (type $B C_{r}$ ) will also appear attached to fine gradings. Following [3] we recall the next definition:

Definition 3.2. Let $\Phi$ be the nonreduced root system $B C_{r}(r \geq 1)$. A Lie algebra $\mathcal{L}$ over $\mathbb{F}$ is graded by $\Phi$, or $\Phi$-graded, if:

(i) $\mathcal{L}$ contains as a subalgebra a finite-dimensional simple Lie algebra $\mathfrak{g}=\mathfrak{h} \oplus$ $\left(\bigoplus_{\alpha \in \Phi^{\prime}} \mathfrak{g}_{\alpha}\right)$ whose root system $\Phi^{\prime}$ relative to a Cartan subalgebra $\mathfrak{h}=\mathfrak{g}_{0}$ is the reduced subsystem of type $B_{r}, C_{r}$, or $D_{r}$ contained in $\Phi$;

(ii) $\mathcal{L}=\bigoplus_{\alpha \in \Phi \cup\{0\}} \mathcal{L}(\alpha)$, where $\mathcal{L}(\alpha)=\{X \in \mathcal{L}:[H, X]=\alpha(H) X$ for all $H \in \mathfrak{h}\}$; and

(iii) $\mathcal{L}(0)=\sum_{\alpha \in \Phi}[\mathcal{L}(\alpha), \mathcal{L}(-\alpha)]$.

Again, the subalgebra $\mathfrak{g}$ is said to be a grading subalgebra of $\mathcal{L}$, and $\mathcal{L}$ is said to be $B C_{r}$-graded with grading subalgebra of type $X_{r}$, where $X_{r}$ is the type of $\mathfrak{g}$.

Only $B C_{r}$-graded subalgebras of type $B_{r}$ will occur in relation to fine gradings on simple Lie algebras.

For $r \geq 3$, let $\mathcal{W}$ be the natural module for the Lie algebra $\mathfrak{g}$ of type $B_{r}$. Thus $\mathcal{W}$ is endowed with a symmetric nondegenerate bilinear form (.|.), and

$$
\begin{aligned}
& \mathfrak{g}=\left\{x \in \operatorname{End}_{\mathbb{F}}(\mathcal{W}):(x u \mid v)=-(u \mid x v) \text { for all } u, v \in \mathcal{W}\right\}, \\
& \mathfrak{s}=\left\{s \in \operatorname{End}_{\mathbb{F}}(\mathcal{W}): \operatorname{trace}(s)=0 \text { and }(s u \mid v)=(u \mid s v) \text { for all } u, v \in \mathcal{W}\right\} .
\end{aligned}
$$

In this case, a $B C_{r}$-graded subalgebra of type $B_{r}$ can be described, up to isomorphism, as follows (see (1.30) in [3]):

$$
\mathcal{L}=(\mathfrak{g} \otimes \mathcal{A}) \oplus(\mathfrak{s} \otimes \mathcal{B}) \oplus(\mathcal{W} \otimes \mathcal{C}) \oplus \mathcal{D},
$$

The bracket in $\mathcal{L}$ gives $\mathfrak{b}=\mathcal{A} \oplus \mathcal{B} \oplus \mathcal{C}$ the structure of an algebra, which is termed the coordinate algebra of $\mathcal{L}$. Moreover (see [3] for details), for $r \geq 3$ we have:

- The sum $\mathfrak{a}=\mathcal{A} \oplus \mathcal{B}$ is a unital associative algebra (the multiplication is denoted by $\alpha \cdot \alpha^{\prime}$ ), with $1 \in \mathcal{A}$ (the subalgebra $\mathfrak{g}$ is identified with $\mathfrak{g} \otimes 1$ ), with involution $\eta$ whose subspace of symmetric elements is $\mathcal{A}$ and whose subspace of skew-symmetric elements is $\mathcal{B}$.

- The space $\mathcal{C}$ is an associative left $\mathfrak{a}$-module (the action is denoted by $\alpha \cdot c$ ), and it is equipped with a hermitian form $\xi$ relative to $\eta$, such that the multiplication in $\mathfrak{b}$ is given by:

$$
(\alpha+c) \cdot\left(\alpha^{\prime}+c^{\prime}\right)=\left(\alpha \cdot \alpha^{\prime}+\xi\left(c, c^{\prime}\right)\right)+\left(\alpha \cdot c^{\prime}+\alpha^{\prime \eta} \cdot c\right) .
$$

For $r=2$, the grading subalgebra $\mathfrak{b}=\mathcal{A} \oplus \mathcal{B} \oplus \mathcal{C}$ is a bit more complicated, and can be described in terms of structurable algebras. (See [3] for details.)

For $r=1$, a $B C_{1}$-graded subalgebra of type $B_{1}$ can be described, up to isomorphism, as follows:

$$
\mathcal{L}=(\mathfrak{g} \otimes \mathcal{A}) \oplus(\mathfrak{s} \otimes \mathcal{B}) \oplus \mathcal{D}
$$


Here the natural module $\mathcal{W}$ for the simple Lie algebra $\mathfrak{g}$ (isomorphic to $\mathfrak{s l}_{2}(\mathbb{F})$ ) of type $B_{1}$ is three-dimensional, and hence isomorphic to the adjoint module $\mathfrak{g}$, and the subspace of symmetric trace-zero endomorphisms $\mathfrak{s}$ is the five-dimensional irreducible module for $\mathfrak{g}$.

In this case, results of Allison [2] give that the coordinate algebra $\mathfrak{a}=\mathcal{A} \oplus \mathcal{B}$ is a structurable algebra whose involution is given by $(a+b)^{\eta}=a-b$ (so $\mathcal{A}$ is the subspace of symmetric elements and $\mathcal{B}$ the subspace of skew-symmetric elements), and the quotient of $\mathcal{L}$ by its center $Z(\mathcal{L})$ is a generalized Tits-Kantor-Koecher Lie algebra constructed from the structurable algebra $(\mathfrak{a}, \eta)$. (See Theorem 2.6 in [7].)

The arguments used in the proof of Theorem 7.5 in [21] give a more precise picture in this situation. The Lie bracket in $\mathcal{L}$, which is invariant under the action of the subalgebra $\mathfrak{g} \simeq \mathfrak{g} \otimes 1$, is given by:

- $\mathcal{D}$ is a subalgebra of $\mathcal{L}$;

- $[A \otimes a, B \otimes b]=[A, B] \otimes a \circ b-\left(A B+B A-\frac{2}{3} \operatorname{trace}(A B) I_{3}\right) \otimes \frac{1}{2}[a, b]+$ $\frac{1}{2} \operatorname{trace}(A B)\langle a \mid b\rangle$;

- $[A \otimes a, X \otimes x]=-(A X+X A) \otimes \frac{1}{2}[a, x]+[A, X] \otimes a \circ x ;$

- $[X \otimes x, Y \otimes y]=[X, Y] \otimes x \circ y-\left(X Y+Y X-\frac{2}{3} \operatorname{trace}(X Y) I_{3}\right) \otimes \frac{1}{2}[x, y]+$ $\frac{1}{2} \operatorname{trace}(X Y)\langle x \mid y\rangle$;

- $[d, A \otimes a]=A \otimes d(a)$;

- $[d, X \otimes x]=X \otimes d(x)$;

for any $A, B \in \mathfrak{g} ; X, Y \in \mathfrak{h} ; a, b \in \mathcal{A} ; x, y \in \mathcal{B}$; and $d \in \mathcal{D}$, where

- $\mathcal{A} \times \mathcal{A} \rightarrow \mathcal{A}:(a, b) \mapsto a \circ b$ is a symmetric bilinear map with $1 \circ a=a$ for any $a \in \mathcal{A}$;

- $\mathcal{A} \times \mathcal{A} \rightarrow \mathcal{B}:(a, b) \mapsto[a, b]$ is a skew symmetric bilinear map with $[1, a]=0$ for any $a \in \mathcal{A}$;

- $\mathcal{A} \times \mathcal{B} \rightarrow \mathcal{A}:(a, x) \mapsto[a, x]$ is a bilinear map with $[1, x]=0$ for any $x \in \mathcal{B}$;

- $\mathcal{A} \times \mathcal{B} \rightarrow \mathcal{B}:(a, x) \mapsto a \circ x$ is a bilinear map with $1 \circ x=x$ for any $x \in \mathcal{B}$;

- $\mathcal{B} \times \mathcal{B} \rightarrow \mathcal{A}:(x, y) \mapsto x \circ y$ is a symmetric bilinear map;

- $\mathcal{B} \times \mathcal{B} \rightarrow \mathcal{B}:(x, y) \mapsto[x, y]$ is a skew symmetric bilinear map;

- $\mathcal{A} \times \mathcal{A} \rightarrow \mathcal{D}:(a, b) \mapsto\langle a \mid b\rangle$ is a skew symmetric bilinear map;

- $\mathcal{B} \times \mathcal{B} \rightarrow \mathcal{D}:(x, y) \mapsto\langle x \mid y\rangle$ is a skew symmetric bilinear map;

- the bilinear maps $\mathcal{D} \times \mathcal{A} \rightarrow \mathcal{A}:(d, a) \mapsto d(a)$ and $\mathcal{D} \times \mathcal{B} \rightarrow \mathcal{B}:(d, x) \mapsto d(x)$, give representations of the Lie algebra $\mathcal{D}$.

Define $x \circ a=a \circ x$ and $[x, a]=-[a, x]$ for any $a \in \mathcal{A}$ and $x \in \mathcal{B}$, and define on the vector space $\mathfrak{a}=\mathcal{A} \oplus \mathcal{B}$ a multiplication by

$$
u \cdot v=u \circ v+\frac{1}{2}[u, v]
$$


for any $u, v \in \mathcal{A} \cup \mathcal{B}$, so $u \circ v=\frac{1}{2}(u \cdot v+v \cdot u)$ and $[u, v]=u \cdot v-v \cdot u$. Define also a linear map $-: \mathfrak{a} \rightarrow \mathfrak{a}$ such that $\overline{a+x}=a-x$ for any $a \in \mathcal{A}$ and $x \in \mathcal{B}$. Then (Theorem 7.5 in [21]) the subspace $\mathfrak{a}$, with this multiplication and involution, is a structurable algebra.

Condition (iii) in Definition 3.2 shows $\mathcal{D}=\langle\mathcal{A} \mid \mathcal{A}\rangle+\langle\mathcal{B} \mid \mathcal{B}\rangle$, and a straightforward application of the Jacobi identity gives

$$
\langle a \mid b\rangle(u)=D_{a, b}(u), \quad\langle x \mid y\rangle(u)=D_{x, y}(u),
$$

for any $a, b \in \mathcal{A}, x, y \in \mathcal{B}$, and $u \in \mathcal{A} \cup \mathcal{B}$, where $D_{u, v}$ is the derivation of the structurable algebra $\mathfrak{a}$ defined in equation (15) in [1]:

$$
D_{u, v}(w)=\frac{1}{3}[[u, v]+[\bar{u}, \bar{v}], w]+(w, v, u)-(w, \bar{u}, \bar{v}),
$$

for $u, v, w \in \mathfrak{a}$, where $(w, v, u)=(w \cdot v) \cdot u-w \cdot(v \cdot u)$ is the associator of the elements $w, v$, and $u$.

\section{Fine gradings on semisimple Lie algebras}

The aim of this section is to show that any fine grading on a finite-dimensional semisimple Lie algebra, with the property that the free rank of its universal group is greater than 0 , determines in a natural way a (possibly nonreduced) root system. This root system is irreducible if the Lie algebra is simple.

The first two items of the next proposition were proved in [13] over the field of complex numbers. Given a finitely generated abelian group $G$, let $\operatorname{tor}(G)$ denote its torsion subgroup and let $\bar{G}$ be the quotient $G / \operatorname{tor}(G)$, which is free. Its rank is called the free rank of $G$.

Proposition 4.1. Let $\mathcal{L}$ be a finite-dimensional semisimple Lie algebra and let $\Gamma: \mathcal{L}=\bigoplus_{g \in G} \mathcal{L}_{g}$ be a fine grading. Assume that $G$ is the universal group of $\Gamma$. (Since the dimension of $\mathcal{L}$ is finite, $G$ is a finitely generated abelian group.)

Then the following conditions hold:

(i) The zero homogeneous component $\mathcal{L}_{0}$ is a toral subalgebra of $\mathcal{L}$ (i.e., ad $\mathcal{L}_{0}$ consists of commuting diagonalizable operators in $\mathcal{L})$.

(ii) The dimension of $\mathcal{L}_{0}$ coincides with the free rank of $G$.

(iii) Let $\operatorname{tor}(G)$ be the torsion subgroup of $G$. The induced grading $\bar{\Gamma}: \mathcal{L}=$ $\bigoplus_{\bar{g} \in G / \operatorname{tor}(G)} \mathcal{L}_{\bar{g}}$ is the weight space decomposition relative to $\mathcal{L}_{0}$.

Proof. The Killing form of $\mathcal{L}$ satisfies $\kappa\left(\mathcal{L}_{g}, \mathcal{L}_{h}\right)=0$ unless $g+h=0$, and hence the restriction of $\kappa$ to $\mathcal{L}_{0}$ is nondegenerate. This shows that $\mathcal{L}_{0}$ is reductive (see Proposition 5 in section 6.4 of chapter I of [10]). Moreover, for any $X \in Z\left(\mathcal{L}_{0}\right)$ (the center of $\left.\mathcal{L}_{0}\right)$, the semisimple and nilpotent parts of $X$ also belong to $Z\left(\mathcal{L}_{0}\right)$ and $\kappa\left(X_{n}, \mathcal{L}_{0}\right)=0$, since $\operatorname{ad} X_{n}$ is nilpotent, so we get $X_{n}=0$. Therefore, the elements 
of $Z\left(\mathcal{L}_{0}\right)$ are semisimple and $\mathcal{L}_{0}$ is reductive in $\mathcal{L}$ (see sections 6.4 and 6.5 in chapter 1 of [10]).

Let $\mathfrak{h}$ be a Cartan subalgebra of $\mathcal{L}_{0}$. Hence $Z\left(\mathcal{L}_{0}\right)$ is contained in $\mathfrak{h}$ and $\mathfrak{h}$ is maximal among the toral subalgebras of $\mathcal{L}$ contained in $\mathcal{L}_{0}$. For any $g \in G, \mathcal{L}_{g}$ is invariant under the adjoint action of $\mathcal{L}_{0}$. Therefore, $\Gamma$ can be refined by means of the weight space decomposition relative to the toral subalgebra $\mathfrak{h}$.

Since $\Gamma$ is fine, for any $g \in G$ there exists a linear form $\alpha \in \mathfrak{h}^{*}$ such that $\mathcal{L}_{g}$ is contained in the weight space

$$
\mathcal{L}(\alpha):=\{X \in \mathcal{L}:[H, X]=\alpha(H) X \text { for all } H \in \mathfrak{h}\}
$$

In particular, $\mathcal{L}_{0}=\mathcal{L}(0) \cap \mathcal{L}_{0}=\mathfrak{h}$ is a toral subalgebra. This proves the first part. (Note that 0 denotes both the zero component of $G$ and the trivial linear form, but this should cause no confusion.)

Therefore, $\Gamma$ is a refinement of the grading given by the weight space decomposition relative to the toral subalgebra $\mathfrak{h}=\mathcal{L}_{0}: \hat{\Gamma}: \mathcal{L}=\bigoplus_{\alpha \in \mathfrak{h}^{*}} \mathcal{L}(\alpha)$. Denote by $\Phi$ the set of nonzero weights in the decomposition

$$
\Phi:=\left\{\alpha \in \mathfrak{h}^{*} \backslash 0: \mathcal{L}(\alpha) \neq 0\right\}
$$

Then $\mathbb{Z} \Phi$ is a free abelian subgroup of $\mathfrak{h}^{*}$ and we can view $\hat{\Gamma}$ as a grading by the group $\mathbb{Z} \Phi$.

Since $G$ is the universal group of $\Gamma$ and $\hat{\Gamma}$ is a coarsening of $\Gamma$, there is a surjective homomorphism

$$
\pi: G \rightarrow \mathbb{Z} \Phi
$$

such that $\pi(g)=\alpha$ if $\mathcal{L}_{g} \subseteq \mathcal{L}(\alpha)$. And since $\mathbb{Z} \Phi$ is torsion free, $\pi$ induces a surjective homomorphism $\bar{\pi}: \bar{G}:=G / \operatorname{tor}(G) \rightarrow \mathbb{Z} \Phi$. In particular, the rank of the free group $\bar{G}$ is greater than or equal to the rank of $\mathbb{Z} \Phi$.

However, $\mathbb{F} \Phi$ is the whole dual vector space $\mathfrak{h}^{*}$, as otherwise there would exist an element $0 \neq X \in \mathfrak{h}$ such that $\alpha(X)=0$ for any $\alpha \in \Phi$, and then $X$ would belong to the center of $\mathcal{L}$, and this is trivial since $\mathcal{L}$ is semisimple. In particular, this shows that the rank of the free abelian group $\mathbb{Z} \Phi$ is greater than or equal to the dimension of the vector space $\mathbb{F} \Phi=\mathfrak{h}^{*}$. Hence we obtain $\operatorname{rank}(\mathbb{Z} \Phi) \geq \operatorname{dim} \mathfrak{h}$, and thus $\operatorname{rank} \bar{G} \geq \operatorname{dim} \mathfrak{h}$.

Since the universal group $G$ is generated by the support of $\Gamma, \bar{G}$ is generated by the support of $\bar{\Gamma}$. But $\bar{G}$ is a finitely generated free abelian group, so there are elements $\bar{g}_{1}, \ldots, \bar{g}_{m} \in \operatorname{Supp} \bar{\Gamma}$ such that $\bar{G}=\mathbb{Z} \bar{g}_{1} \oplus \cdots \oplus \mathbb{Z} \bar{g}_{m}$ (here $\bar{g}$ denotes the class of $g$ modulo tor $(G))$.

The Lie algebra $\mathcal{L}$ is semisimple, and hence any derivation is inner. In particular, for any $i=1, \ldots, m$, there is a unique element $H_{i} \in \mathcal{L}$ such that $\left[H_{i}, X\right]=n_{i} X$ for any $X \in \mathcal{L}_{n_{1} \bar{g}_{1}+\cdots+n_{m} \bar{g}_{m}}$. Moreover, we may replace $H_{i}$ by its component in $\mathcal{L}_{0}=\mathfrak{h}$ for any $i$, so, by uniqueness, we obtain $H_{1}, \ldots, H_{m} \in \mathcal{L}_{0}$. Since the sum $\mathcal{L}_{\bar{g}_{1}} \oplus \cdots \oplus \mathcal{L}_{\bar{g}_{m}}$ is direct, the elements $H_{1}, \ldots, H_{m}$ are linearly independent, and hence we get $m=\operatorname{rank} \bar{G} \leq \operatorname{dim} \mathfrak{h}$. This proves the second part: $\operatorname{rank} \bar{G}=\operatorname{dim} \mathfrak{h}$. 
The argument above shows that $\mathfrak{h}=\mathbb{F} H_{1} \oplus \cdots \oplus \mathbb{F} H_{m}$, and for any $\bar{g}=$ $n_{1} \bar{g}_{1}+\cdots+n_{m} \bar{g}_{m}$ we have $\mathcal{L}_{\bar{g}}=\mathcal{L}(\alpha)$, where $\alpha$ is the linear form on $\mathfrak{h}$ such that $\alpha\left(H_{i}\right)=n_{i}$ for any $i$. This proves the last part.

Remark 4.2. The zero component of the grading $\bar{\Gamma}$ in Proposition 4.1 is $\mathcal{L}_{\overline{0}}=$ $\bigoplus_{g \in \operatorname{tor}(G)} \mathcal{L}_{g}=\mathcal{L}(0)$, and this is the centralizer $\operatorname{Cent}_{\mathcal{L}}\left(\mathcal{L}_{0}\right)$.

Using the arguments in the proof above, $\mathcal{L}(0)$ is shown to be reductive in $\mathcal{L}$, so $\mathcal{L}(0)=Z(\mathcal{L}(0)) \oplus[\mathcal{L}(0), \mathcal{L}(0)]$ and $\mathcal{L}_{0} \subseteq Z(\mathcal{L}(0))$. In particular, the zero component of the restriction of $\Gamma$ to $[\mathcal{L}(0), \mathcal{L}(0)]$ is trivial: $[\mathcal{L}(0), \mathcal{L}(0)]_{0}=0$.

Therefore, $\Gamma$ induces a grading on $[\mathcal{L}(0), \mathcal{L}(0)]$ by the finite group $\operatorname{tor}(G)$ whose homogeneous component of degree 0 is trivial. These gradings are called special. (See [23] for properties of these gradings.)

Remark 4.3. Condition (ii) in Proposition 4.1 does not suffice to ensure that the grading $\Gamma$ is fine. As an example, let $V$ be a five-dimensional vector space with a basis $\left\{e_{1}, e_{2}, e_{3}, e_{4}, e_{5}\right\}$, endowed with the symmetric bilinear form $b$ such that $b\left(e_{i}, e_{j}\right)=\delta_{i j}$ for any $i$ and $j$. Consider the associated orthogonal Lie algebra $\mathfrak{s o}(V, b)$ comprising endomorphisms skew-symmetric with respect to $b$. The vector space $V$ is graded by $\mathbb{Z}_{2}^{3}$, with $\operatorname{deg} e_{1}=(\overline{1}, \overline{0}, \overline{0}), \operatorname{deg} e_{2}=(\overline{0}, \overline{1}, \overline{0}), \operatorname{deg} e_{3}=(\overline{0}, \overline{0}, \overline{1})$, $\operatorname{deg} e_{4}=(\overline{1}, \overline{1}, \overline{1})$, and $\operatorname{deg} e_{5}=0$. This induces a grading by $\mathbb{Z}_{2}^{3}$ on $\mathfrak{s o}(V, b)$, of type $(4,3)$, with the basic elements $E_{i j}-E_{j i}, i \neq j$, being homogeneous of degree $\operatorname{deg} e_{i}+\operatorname{deg} e_{j}$. Here $E_{i j}$ denotes the endomorphism that takes $e_{j}$ to $e_{i}$ and annihilates the other basic elements. Then $\mathfrak{s l}(V, b)_{0}=0$, the free rank of the finite grading group is also 0 , but this grading is not fine, as it can be refined to get a grading of type $(10)$ by $\mathbb{Z}_{2}^{4}$ with $\operatorname{deg} e_{1}=(\overline{1}, \overline{0}, \overline{0}, \overline{0}), \operatorname{deg} e_{2}=(\overline{0}, \overline{1}, \overline{0}, \overline{0})$, $\operatorname{deg} e_{3}=(\overline{0}, \overline{0}, \overline{1}, \overline{0}), \operatorname{deg} e_{4}=(\overline{0}, \overline{0}, \overline{0}, \overline{1})$, and $\operatorname{deg} e_{5}=0$.

Theorem 4.4. Let $\mathcal{L}$ be a finite dimensional semisimple Lie algebra and let $\Gamma: \mathcal{L}=\bigoplus_{g \in G} \mathcal{L}_{g}$ be a fine grading. Assume that $G$ is the universal group of $\Gamma$. Let $\Phi$ be as in (4.1). Then, $\Phi$ is a (possibly nonreduced) root system in the Euclidean vector space $E=\mathbb{R} \otimes_{\mathbb{Q}} \mathbb{Q} \Phi$. If $\mathcal{L}$ is simple, then $\Phi$ is an irreducible root system.

Proof. The proof requires several steps:

1. By Proposition 4.1, the set of weights $\Phi$ is precisely $\pi(\operatorname{Supp} \Gamma \backslash \operatorname{tor}(G))$, with $\pi$ in (4.2). For any $g \in \operatorname{Supp} \Gamma \backslash \operatorname{tor}(G)$, let $\alpha=\pi(g)$ and take an element $0 \neq X \in \mathcal{L}_{g} \subseteq \mathcal{L}(\alpha)$. Then $\mathcal{L}_{-g}$ is contained in $\mathcal{L}(-\alpha)$. Since $\alpha$ is not $0, \operatorname{ad} X$ is nilpotent. By the Jacobson-Morozov Theorem (see Theorem 17 in Chapter III of $[26])$, there are elements $H, Y \in \mathcal{L}$ such that $[H, X]=2 X,[H, Y]=-2 Y$ and $[X, Y]=H$ (i.e., $X, H, Y$ form an $\mathfrak{s l}_{2}$-triple). We have $H=\sum_{h \in G} H_{h}$ and $Y=\sum_{h \in G} Y_{h}$ for homogeneous elements $H_{h}, Y_{h} \in \mathcal{L}_{h}, h \in G$. Then $[H, X]=2 X$ implies $\left[H_{0}, X\right]=2 X$, so $\alpha\left(H_{0}\right)=2$, and hence $\left[H_{0}, Y_{-g}\right]=-2 Y_{-g}$. Also, from $[X, Y]=H$ we get $\left[X, Y_{-g}\right]=H_{0}$. Therefore, we may take $H \in \mathcal{L}_{0}=\mathfrak{h}$ and $Y \in \mathcal{L}_{-g}$.

2. The restriction of the Killing form $\kappa$ to $\mathfrak{h}=\mathcal{L}_{0}$ is nondegenerate, so it induces a nondegenerate symmetric bilinear form $\left(. \mid\right.$.) on $\mathfrak{h}^{*}=\mathbb{F} \Phi$. For any $\alpha \in \Phi$, take 
an element $g \in G$ with $\pi(g)=\alpha$, and an $\mathfrak{s l}_{2}$-triple $X \in \mathcal{L}_{g}, H \in \mathcal{L}_{0}$, and $Y \in \mathcal{L}_{-g}$ as above. For any $\beta \in \Phi$, the sum $\bigoplus_{i \in \mathbb{Z}} \mathcal{L}(\beta+i \alpha)$ is a module for the subalgebra $\mathfrak{s}=\operatorname{span}\{X, H, Y\}$ (isomorphic to $\mathfrak{s l}_{2}(\mathbb{F})$ ). With standard arguments we obtain $\beta(H)=r-q \in \mathbb{Z}$ and $\beta-\beta(H) \alpha \in \Phi$, where $q=\max \{n \in \mathbb{Z}: \beta+n \alpha \in \Phi\}$, $r=\max \{n \in \mathbb{Z}: \beta-n \alpha \in \Phi\}$. In particular, $H_{\alpha}:=H$ does not depend on $g$ or $X$, only on $\alpha$. Also, we get

$$
\kappa\left(H_{\alpha}, H_{\alpha}\right)=\sum_{\beta \in \Phi}(\operatorname{dim} \mathcal{L}(\beta)) \beta\left(H_{\alpha}\right)^{2} \in \mathbb{Z}_{>0} .
$$

3. For any $\alpha \in \mathfrak{h}^{*}$ there is a unique $T_{\alpha} \in \mathfrak{h}$ such that $\alpha(H)=\kappa\left(T_{\alpha}, H\right)$ for any $H \in \mathfrak{h}$. If the element $T \in \mathfrak{h}=\mathcal{L}_{0}$ satisfies $\kappa\left(T_{\alpha}, T\right)=\alpha(T)=0$, then for any $\beta \in \Phi$ we have

$$
\operatorname{trace}\left(\left.\left(\operatorname{ad} H_{\alpha} \operatorname{ad} T\right)\right|_{\bigoplus_{i \in \mathbb{Z}} \mathcal{L}_{\beta+i \alpha}}\right)=\beta(T) \operatorname{trace}\left(\left.\operatorname{ad} H_{\alpha}\right|_{\oplus_{i \in \mathbb{Z}} \mathcal{L}_{\beta+i \alpha}}\right)=0 .
$$

Consequently, $\kappa\left(H_{\alpha}, T\right)=0$ also, and hence $H_{\alpha} \in \mathbb{F} T_{\alpha}$. Since $\alpha\left(H_{\alpha}\right)=2$, we get

$$
H_{\alpha}=\frac{2}{\kappa\left(T_{\alpha}, T_{\alpha}\right)} T_{\alpha}=\frac{2}{(\alpha \mid \alpha)} T_{\alpha} .
$$

Define, as usual,

$$
\langle\beta \mid \alpha\rangle:=\frac{2(\beta \mid \alpha)}{(\alpha \mid \alpha)}=\beta\left(H_{\alpha}\right) .
$$

Therefore we have for any $\alpha, \beta \in \Phi$ that

$$
\langle\beta \mid \alpha\rangle \in \mathbb{Z} \text { and } \beta-\langle\beta \mid \alpha\rangle \alpha \in \Phi .
$$

Also we have $\kappa\left(H_{\alpha}, H_{\alpha}\right)=\frac{4}{(\alpha \mid \alpha)^{2}} \kappa\left(T_{\alpha}, T_{\alpha}\right)=\frac{4}{(\alpha \mid \alpha)}$, so $(\alpha \mid \alpha)=\frac{4}{\kappa\left(H_{\alpha}, H_{\alpha}\right)}$ is a positive rational number.

4. Take a basis $\left\{\alpha_{1}, \ldots, \alpha_{m}\right\}$ of $\mathfrak{h}^{*}$ contained in $\Phi$, and let $g_{1}, \ldots, g_{m}$ be elements of $G$ such that $\pi\left(g_{i}\right)=\alpha_{i}$ for any $i=1, \ldots, m$. For any $\gamma \in \mathbb{Q} \Phi\left(\subseteq \mathfrak{h}^{*}\right)$, there are rational numbers $r_{1}, \ldots, r_{m}$ such that $\gamma=r_{1} \alpha_{1}+\cdots+r_{m} \alpha_{m}$, and we get

$$
\begin{aligned}
(\gamma \mid \gamma) & =\kappa\left(T_{\gamma}, T_{\gamma}\right)=\sum_{\beta \in \Phi}(\operatorname{dim} \mathcal{L}(\beta)) \beta\left(T_{\gamma}\right)^{2} \\
& =\sum_{\beta \in \Phi}(\operatorname{dim} \mathcal{L}(\beta))\left(\sum_{i=1}^{m} r_{i} \beta\left(T_{\alpha_{i}}\right)^{2}=\sum_{\beta \in \Phi}(\operatorname{dim} \mathcal{L}(\beta))\left(\sum_{i=1}^{m} r_{i}\left(\beta \mid \alpha_{i}\right)\right)^{2}\right. \\
& =\sum_{\beta \in \Phi}(\operatorname{dim} \mathcal{L}(\beta))\left(\sum_{i=1}^{m} \frac{r_{i}\left(\alpha_{i} \mid \alpha_{i}\right)}{2}\left\langle\beta \mid \alpha_{i}\right\rangle\right)^{2} \in \mathbb{Q}_{>0} .
\end{aligned}
$$

Hence $E=\mathbb{R} \otimes_{\mathbb{Q}} \mathbb{Q} \Phi$ is a Euclidean vector space with inner product determined by $(. \mid.) ; \Phi$ is a finite subset of $E$ not containing 0 , that spans $E$ and such that $\langle\alpha \mid \beta\rangle=\frac{2(\alpha \mid \beta)}{(\beta \mid \beta)} \in \mathbb{Z}$; and $\beta-\langle\beta \mid \alpha\rangle \alpha \in \Phi$, for any $\alpha, \beta \in \Phi$. Therefore, $\Phi$ is a root system.

5. If $\mathcal{L}$ is simple, then $\Phi$ must be irreducible, as otherwise $\Phi$ would split as a disjoint union $\Phi=\Phi_{1} \cup \dot{\cup} \Phi_{2}$, with $\left(\Phi_{1} \mid \Phi_{2}\right)=0$. But then $\left(\bigoplus_{\alpha \in \Phi_{1}} \mathcal{L}(\alpha)\right) \oplus$ $\left(\sum_{\alpha \in \Phi_{1}}[\mathcal{L}(\alpha), \mathcal{L}(-\alpha)]\right)$ would be a proper ideal of $\mathcal{L}$. 


\section{The main result}

With the same hypotheses as in the previous section, take a system of simple roots $\Delta$ of the root system $\Phi$ in (4.1). Hence $\Delta$ is a basis of $\mathfrak{h}^{*}$ contained in $\Phi$ and $\Phi=\Phi^{+} \dot{\cup} \Phi^{-}$, with $\Phi^{+} \subseteq \sum_{\alpha \in \Delta} \mathbb{Z}_{\geq 0} \alpha, \Phi^{-}=-\Phi^{+}$. For any $\alpha \in \Delta$ choose $g_{\alpha} \in G$ such that $\pi\left(g_{\alpha}\right)=\alpha$. Since $G$ is generated by Supp $\Gamma$, we have

$$
G=\left(\bigoplus_{\alpha \in \Delta} \mathbb{Z} g_{\alpha}\right) \oplus \operatorname{tor}(G)
$$

Let

$$
\tilde{G}:=\bigoplus_{\alpha \in \Delta} \mathbb{Z} g_{\alpha}
$$

and let

$$
\mathfrak{g}:=\bigoplus_{g \in \tilde{G}} \mathcal{L}_{g} .
$$

The arguments in the proof of Proposition 4.1 show that $\mathfrak{g}$ is a reductive subalgebra of $\mathcal{L}$. Also, any $0 \neq X \in \mathfrak{g}_{g}, g \neq 0$, is contained in a $\mathfrak{s l}_{2}$-triple, so the center $Z(\mathfrak{g})$ is contained in $\mathcal{L}_{0}=\mathfrak{h}$. As the dimension of $\mathfrak{h}$ coincides with the rank of $\mathbb{Z} \Phi$, we conclude that $Z(\mathfrak{g})=0$ and $\mathfrak{g}$ is semisimple.

Also, any weight of $\mathfrak{h}$ on $\mathfrak{g}$ belongs to $\pm\left(\bigoplus_{\alpha \in \Delta} \mathbb{Z}_{\geq 0} \alpha\right)$, so $\Delta$ is a system of simple roots for $\mathfrak{g}$ relative to its Cartan subalgebra $\mathfrak{h}$. We conclude that $\mathfrak{g}$ is, up to isomorphism, the semisimple Lie algebra with $\Delta$ as a system of simple roots.

Now the main result of the paper, relating fine gradings and gradings by root systems, follows easily.

Theorem 5.1. Let $\mathcal{L}$ be a finite dimensional simple Lie algebra and let $\Gamma: \mathcal{L}=$ $\bigoplus_{g \in G} \mathcal{L}_{g}$ be a fine grading. Assume that $G$ is the universal group of $\Gamma$. Let $\Phi$ be as in (4.1). Then $\mathcal{L}$ is graded by the irreducible (possibly nonreduced) root system $\Phi$ with grading subalgebra $\mathfrak{g}$ as in (5.1). Moreover, if $\Phi$ is nonreduced (type $B C_{r}$ ), then $\mathfrak{g}$ is simple of type $B_{r}$.

Proof. The Lie algebra $\mathcal{L}$ contains the semisimple subalgebra $\mathfrak{g}$ with Cartan subalgebra $\mathfrak{h}$ and system of simple roots $\Delta$. Since $\mathcal{L}$ is simple, $\Phi$ (or $\Delta$ ) is irreducible, and the ideal $\left(\bigoplus_{\alpha \in \Phi} \mathcal{L}(\alpha)\right) \oplus\left(\sum_{\alpha \in \Phi}[\mathcal{L}(\alpha), \mathcal{L}(-\alpha)]\right)$ is all of $\mathcal{L}$. Hence $\mathcal{L}$ is graded by the root system $\Phi$ with $\mathfrak{g}$ as a grading subalgebra. Moreover, any root in $\Phi$ is a sum of roots in $\mathfrak{g}$. Hence for $\Phi$ of type $B C_{r}, \mathfrak{g}$ is of type $B_{r}$.

\section{Grading on the coordinate algebra}

Let $\Gamma: \mathcal{L}=\bigoplus_{g \in G} \mathcal{L}_{g}$ be a fine grading on a finite-dimensional simple Lie algebra, with $G$ being the universal group of $\Gamma$. As in the proof of Proposition 4.1, let $\Phi$ be the set of weights of the adjoint action of $\mathcal{L}_{0}$, and let $\pi: G \rightarrow \mathbb{Z} \Phi$ be the surjective group homomorphism with $\pi(g)=\alpha$ if $\mathcal{L}_{g} \subseteq \mathcal{L}(\alpha)$. Then $\pi$ induces an 
isomorphism $\bar{\pi}: \bar{G}=G / \operatorname{tor}(G) \rightarrow \mathbb{Z} \Phi$ by item (iii) of Proposition 4.1. Let $\mathfrak{g}$ be the grading subalgebra in Theorem 5.1, obtained after fixing a system of simple roots $\Delta$ and preimages $g_{\alpha}$ under $\pi$ of the elements in $\Delta$. Also, consider the free abelian group $\tilde{G}=\bigoplus_{\alpha \in \Delta} \mathbb{Z} g_{\alpha}$, such that $G=\tilde{G} \oplus \operatorname{tor}(G)$. The restriction of $\pi$ to $\tilde{G}$ is bijective.

If $\Phi$ is reduced, then we have a decomposition as in equation (3.1). Then:

- $\mathfrak{g}=\mathfrak{g} \otimes 1$ is, by its construction, a graded subalgebra of $\mathcal{L}$, and hence so is its centralizer $\mathcal{D}=\operatorname{Cent}_{\mathcal{L}}(\mathfrak{g})$. Moreover, $\mathcal{D}$ is contained in $\mathcal{L}(0)=\bigoplus_{g \in \operatorname{tor}(G)} \mathcal{L}_{g}$, and hence $\mathcal{D}$ is graded by $\operatorname{tor}(G)$. Moreover, $\mathcal{D}_{0} \subseteq \mathcal{L}_{0}=\mathfrak{g}_{0}$, so $\mathcal{D}_{0}=0$. Therefore, the grading of $\mathcal{D}$ by $\operatorname{tor}(G)$ is a special grading. (See Remark 4.2.)

- Let $\lambda$ be the highest root of $\mathfrak{g}$ (relative to $\Delta$ ), then $\lambda$ is not a weight of $\mathcal{W}$, and hence $\mathcal{L}(\lambda)=\mathfrak{g}_{\lambda} \otimes \mathcal{A}$. On the other hand, if $g_{\lambda}$ is the preimage in $\tilde{G}$ of $\lambda$, then

$$
\mathcal{L}(\lambda)=\bigoplus\left\{\mathcal{L}_{g}: \pi(g)=\lambda\right\}=\bigoplus_{g \in \operatorname{tor}(G)} \mathcal{L}_{g_{\lambda}+g},
$$

so the vector space $\mathcal{A}$ is graded by $\operatorname{tor}(G)$, where $\mathcal{A}_{h}$ is defined by

$$
\mathcal{L}_{g_{\lambda}+h}=\mathfrak{g}_{\lambda} \otimes \mathcal{A}_{h}
$$

for any $h \in \operatorname{tor}(G)$.

- Since $\mathfrak{g} \otimes \mathcal{A}$ is the $\mathfrak{g}$-submodule of $\mathcal{L}$ generated by $\mathfrak{g}_{\lambda} \otimes \mathcal{A}(\lambda$ is the highest root of $\mathfrak{g})$, it follows that $\mathfrak{g} \otimes \mathcal{A}$ is a graded subspace of $\mathcal{L}$ and for any $g \in \tilde{G}$ and $h \in \operatorname{tor}(G)$ we have

$$
(\mathfrak{g} \otimes \mathcal{A})_{g+h}=\mathfrak{g}_{\pi(g)} \otimes \mathcal{A}_{h}
$$

- By invariance under the adjoint action of $\mathfrak{g}$, the subspace $\mathcal{W} \otimes \mathcal{B}$ is the orthogonal complement of $(\mathfrak{g} \otimes \mathcal{A}) \oplus \mathcal{D}$ relative to the Killing form of $\mathcal{L}$. Since this subspace is a graded subspace of $\mathcal{L}$, so is $\mathcal{W} \otimes \mathcal{B}$.

Let $\mu$ be the highest weight of the $\mathfrak{g}$-module $\mathcal{W}$ relative to $\Delta(\mu$ is the highest short root). Let $g_{\mu}$ be the preimage under $\pi$ of $\mu$ in $\tilde{G}$. Then, as for $\mathcal{A}$, we also get that $\mathcal{B}$ is graded by $\operatorname{tor}(G)$ if we define $\mathcal{B}_{h}$ by

$$
(\mathcal{W} \otimes \mathcal{B})_{g_{\mu}+h}=\mathcal{W}_{\mu} \otimes \mathcal{B}_{h},
$$

for any $h \in \operatorname{tor}(G)$. Since $\mathcal{W}$ is generated, as a module for $\mathfrak{g}$, by $\mathcal{W}_{\mu}(=\{w \in$ $\mathcal{W}: H . w=\mu(H) w$ for all $\left.\left.H \in \mathfrak{h}=\mathfrak{g}_{0}\right\}\right)$, it follows that the subspace $\mathcal{W} \otimes \mathcal{B}$ is a graded subspace of $\mathcal{L}$ and, for any $g \in \tilde{G}$ and $h \in \operatorname{tor}(G)$, we have

$$
(\mathcal{W} \otimes \mathcal{B})_{g+h}=\mathcal{W}_{\pi(g)} \otimes \mathcal{B}_{h}
$$

On the other hand, if $\Phi$ is nonreduced of type $B C_{1}$, then we have a decomposition as in equation (3.4), and the same arguments show that $\mathcal{D}$ inherits a special grading by $\operatorname{tor}(G)$, that if $\mu$ is the highest weight then $\mathcal{L}(\mu)=\mathcal{W}_{\mu} \otimes \mathcal{B}$, and this 
shows that $\mathcal{B}$ is graded by $\operatorname{tor}(G)$ as above. Finally, $\mathfrak{g} \otimes \mathcal{A}$ is the orthogonal complement to $(\mathcal{W} \otimes \mathcal{B}) \oplus \mathcal{D}$ relative to the Killing form, and we conclude that $\mathcal{A}$ is graded also by $\operatorname{tor}(G)$ as above.

Finally, if $\Phi$ is nonreduced of type $B C_{r}, r \geq 2$, then we have a decomposition as in equation (3.3) and one checks as before that $\mathcal{D}$ inherits a special grading by $\operatorname{tor}(G)$, that if $\mu$ is the highest weight of $\mathfrak{s}$, then $\mathcal{L}(\mu)=\mathfrak{s}_{\mu} \otimes \mathcal{B}$, and hence it follows that $\mathcal{B}$ is $\operatorname{tor}(G)$-graded. Then $(\mathfrak{g} \otimes \mathcal{A}) \oplus(\mathcal{W} \otimes \mathcal{C})$ is the orthogonal complement of $(\mathfrak{s} \otimes \mathcal{B}) \oplus \mathcal{D}$, so it is a graded subspace too. Here, if $\lambda$ is the highest root, then $((\mathfrak{g} \otimes \mathcal{A}) \oplus(\mathcal{W} \otimes \mathcal{C})) \cap \mathcal{L}(\lambda)=\mathfrak{g}_{\lambda} \otimes \mathcal{A}$, so again we conclude that $\mathcal{A}$ is $\operatorname{tor}(G)$-graded, and from this we deduce that so is $\mathcal{C}$.

These arguments prove most of the next result.

Proposition 6.1. Under the conditions above, with $\Phi$ being an irreducible root system, the coordinate algebra $\mathfrak{a}=\mathcal{A} \oplus \mathcal{B}$ (in the reduced case or for $B C_{1}$ ) or $\mathfrak{b}=\mathfrak{a} \oplus \mathcal{C}$ (in the $B C_{r}$-case, $r \geq 2$ ) inherits a fine grading by $\operatorname{tor}(G)$, where $\mathcal{A}$ and $\mathcal{B}$, and $\mathcal{C}$ in the $B C_{r}$-case, $r \geq 2$, are graded subspaces.

Moreover, $\mathcal{A}_{0}=\mathbb{F} 1$ while $\mathcal{B}_{0}=0$, and also $\mathcal{C}_{0}=0$ (in the $B C_{r}$-case, $r \geq 2$ ), $\operatorname{tor}(G)$ is the universal group, and this grading on $\mathfrak{a}$, or $\mathfrak{b}$, induces a special grading on $\mathcal{D}$ by $\operatorname{tor}(G)$.

Proof. The fact that $\mathfrak{a}$ inherits a grading by $\operatorname{tor}(G)$ is clear from the earlier arguments. Also $\mathcal{L}_{0}=\mathfrak{g}_{0}=\mathfrak{g}_{0} \otimes 1$, so $\mathcal{A}_{0}=\mathbb{F} 1$ and $\mathcal{B}_{0}=0$ (and $\mathcal{C}_{0}=0$ too in the $B C_{r}$ case, $r \geq 2$ ). Hence $\mathfrak{a}_{0}=\mathbb{F} 1$. Moreover, any refinement of this grading on $\mathfrak{a}$ would give a refinement of $\Gamma$, as the grading by $\operatorname{tor}(G)$ of $\mathcal{D}$ is determined by the grading on $\mathfrak{a}$, because of condition (iii) in Definition 3.1. The last part is a direct consequence of $G$ being the universal group of $\Gamma$.

\section{Applications}

The results in the previous sections will be used to classify the fine gradings on the simple exceptional Lie algebras whose universal groups have free rank $>2$. Quick proofs of the classification of fine gradings, up to equivalence, on the simple Lie algebras of types $G_{2}$ and $F_{4}$ will be given too.

Table 25 in [15] gives a list of the simple subalgebras of rank at least two of the exceptional simple Lie algebras, together with the decomposition of any such simple Lie algebra as a sum of irreducible modules for the simple subalgebra. This immediately gives the different possibilities, up to conjugation, for the gradings of an exceptional simple Lie algebra by an irreducible (not necessarily reduced) root system of rank at least two. The different possibilities are summarized in Table 1 , where $\mathfrak{g}, \mathfrak{s}, \mathcal{W}, \mathcal{A}, \mathcal{B}, \mathcal{C}$, and $\mathcal{D}$ are as in equations (3.1) or (3.3).

In many cases, this corresponds (see [8] and [29]) to the well-known Tits construction $\mathcal{T}(\mathcal{C}, \mathcal{J})$, for a unital composition algebra $\mathcal{C}$ and a degree three simple Jordan algebra $\mathcal{J}$, which we recall now (see also [21]).

Let $\mathcal{H}$ be a unital composition algebra (or Hurwitz algebra) with norm $n$ and trace $t$. The unital composition algebras are, up to isomorphism, $\mathbb{F}, \mathbb{K}=\mathbb{F} \oplus \mathbb{F}$, 
$\mathbb{H}=\operatorname{Mat}_{2}(\mathbb{F})$ (the quaternion algebra), and the Cayley algebra $\mathbb{O}$ (recall that the ground field $\mathbb{F}$ is assumed to be algebraically closed). Let $\mathcal{J}$ be a unital simple Jordan algebra of degree 3 , so that $\mathcal{J}$ is the Jordan algebra $H_{3}\left(\mathcal{H}^{\prime}\right)$ of hermitian $3 \times 3$ matrices over another unital composition algebra $\mathcal{H}^{\prime}$. Denote by $\mathcal{H}_{0}$ and $\mathcal{J}_{0}$ the subspaces of trace zero elements in $\mathcal{H}$ and $\mathcal{J}$.

For $a, b \in \mathcal{H}$, the linear map $D_{a, b}: \mathcal{H} \rightarrow \mathcal{H}$ defined by

$$
D_{a, b}(c)=[[a, b], c]+3(a, c, b),
$$

where $[a, b]=a b-b a$ is the commutator, and $(a, c, b)=(a c) b-a(c b)$ is the associator, is a derivation of $\mathcal{H}$. These derivations span the Lie algebra $\operatorname{Der}(\mathcal{H})$.

Similarly, for $x, y \in \mathcal{J}$, the linear map $d_{x, y}: \mathcal{J} \rightarrow \mathcal{J}$ defined by

$$
d_{x, y}(z)=x(y z)-y(x z)
$$

is the inner derivation of $\mathcal{J}$ determined by the elements $x$ and $y$. These derivations span the Lie algebra $\operatorname{Der}(\mathcal{J})$ of derivations.

Given $\mathcal{H}$ and $\mathcal{J}$ as before, consider the space

$$
\mathcal{T}(\mathcal{H}, \mathcal{J})=\operatorname{Der}(\mathcal{H}) \oplus\left(\mathcal{H}_{0} \otimes \mathcal{J}_{0}\right) \oplus \operatorname{Der}(\mathcal{J}),
$$

with the anticommutative multiplication [., .] specified by:

- $\operatorname{Der}(\mathcal{H})$ and $\operatorname{Der}(\mathcal{J})$ are Lie subalgebras, and $[\operatorname{Der}(\mathcal{H}), \operatorname{Der}(\mathcal{J})]=0$;

- $[D, a \otimes x]=D(a) \otimes x,[d, a \otimes x]=a \otimes d(x)$;

- $[a \otimes x, b \otimes y]=\operatorname{trace}(x y) D_{a, b}+([a, b] \otimes x * y)+2 t(a b) d_{x, y} ;$

for all $D \in \operatorname{Der}(\mathcal{H}), d \in \operatorname{Der}(\mathcal{J}), a, b \in \mathcal{H}_{0}$, and $x, y \in \mathcal{J}_{0}$, where

$$
x * y=x y-\frac{1}{3} \operatorname{trace}(x y) 1 .
$$

Looking at equation (7.2) from the left, in the case $\mathcal{H}$ is the Cayley algebra $\mathbb{O}$ (i.e., $\operatorname{dim} \mathcal{H}=8$ ), then $\operatorname{Der}(\mathbb{O})$ is the simple Lie algebra of type $G_{2}$ and (7.2) gives a decomposition as in equation (3.1), thus proving that $\mathcal{T}(\mathbb{O}, \mathcal{J})$ is graded by the root system of type $G_{2}$ with coordinate algebra $\mathcal{J}=\mathbb{F} 1 \oplus \mathcal{J}_{0}$.

Looking at equation (7.2) from the right, we obtain:

- If $\mathcal{J}$ is the Albert algebra $\mathbb{A}$ (i.e., $\mathcal{J}$ is the algebra of hermitian $3 \times 3$-matrices over the Cayley algebra), then $\operatorname{Der}(\mathbb{A})$ is the simple Lie algebra of type $F_{4}$, and (7.2) proves that $\mathcal{T}(\mathcal{H}, \mathbb{A})$ is graded by the root system of type $F_{4}$ with coordinate algebra $\mathcal{H}=\mathbb{F} 1 \oplus \mathcal{H}_{0}$.

- If $\mathcal{J}$ is the Jordan algebra $H_{3}(\mathbb{H})$, then $\operatorname{Der}(\mathcal{J})$ is the simple Lie algebra of type $C_{3}$, and $\mathcal{T}(\mathcal{H}, \mathcal{J})$ is graded by the root system of type $C_{3}$ with coordinate algebra $\mathcal{H}$.

- If $\mathcal{J}$ is the Jordan algebra $\operatorname{Mat}_{3}(\mathbb{F})^{+}=H_{3}(\mathbb{K})$, then $\operatorname{Der}(\mathcal{J})$ is the simple Lie algebra of type $A_{2}$, and then $\mathcal{T}(\mathcal{H}, \mathcal{J})$ is graded by the root system of type $A_{2}$ with coordinate algebra $\mathcal{H}$. 


\begin{tabular}{|c|c|c|c|c|c|c|c|}
\hline $\begin{array}{c}\text { Lie } \\
\text { algebra }\end{array}$ & $\begin{array}{c}\text { Root } \\
\text { system }\end{array}$ & $\operatorname{dim} \mathcal{A}$ & $\operatorname{dim} \mathcal{B}$ & $\operatorname{dim} \mathcal{C}$ & $\operatorname{dim} \mathcal{D}$ & model & $\begin{array}{c}\text { coordinate } \\
\text { algebra }\end{array}$ \\
\hline \hline$G_{2}$ & $G_{2}$ & 1 & 0 & - & 0 & & $\mathbb{F}$ \\
\hline \hline$F_{4}$ & $G_{2}$ & 1 & 5 & - & 3 & $\mathcal{T}\left(\mathbb{O}, H_{3}(\mathbb{F})\right)$ & $H_{3}(\mathbb{F})$ \\
\hline$F_{4}$ & $F_{4}$ & 1 & 0 & - & 0 & & $\mathbb{F}$ \\
\hline \hline$E_{6}$ & $A_{2}$ & 8 & - & - & 14 & $\mathcal{T}\left(\mathbb{O}, H_{3}(\mathbb{K})\right)$ & $\mathbb{O}$ \\
\hline$E_{6}$ & $B C_{2}$ & 5 & 1 & 2 & 4 & & \\
\hline$E_{6}$ & $G_{2}$ & 1 & 8 & - & 8 & $\mathcal{T}\left(\mathbb{O}, H_{3}(\mathbb{K})\right)$ & $\operatorname{Mat}_{3}(\mathbb{F})^{+}$ \\
\hline$E_{6}$ & $F_{4}$ & 1 & 1 & - & 0 & $\mathcal{T}(\mathbb{K}, \mathbb{A})$ & $\mathbb{K}$ \\
\hline$E_{6}$ & $E_{6}$ & 1 & 0 & - & 0 & & $\mathbb{F}$ \\
\hline \hline$E_{7}$ & $B C_{2}$ & 7 & 1 & 8 & 9 & & \\
\hline$E_{7}$ & $G_{2}$ & 1 & 14 & - & 21 & $\mathcal{T}\left(\mathbb{O}, H_{3}(\mathbb{H})\right)$ & $H_{3}(\mathbb{H})$ \\
\hline$E_{7}$ & $C_{3}$ & 1 & 7 & - & 14 & $\mathcal{T}\left(\mathbb{O}, H_{3}(\mathbb{H})\right)$ & $\mathbb{O}$ \\
\hline$E_{7}$ & $F_{4}$ & 1 & 3 & - & 3 & $\mathcal{T}(\mathbb{H}, \mathbb{A})$ & $\mathbb{H}$ \\
\hline$E_{7}$ & $E_{7}$ & 1 & 0 & - & 0 & & $\mathbb{F}$ \\
\hline \hline$E_{8}$ & $B_{2}$ & 11 & 1 & 20 & 24 & & \\
\hline$E_{8}$ & $G_{2}$ & 1 & 26 & - & 52 & $\mathcal{T}(\mathbb{O}, \mathbb{A})$ & $\mathbb{A}$ \\
\hline$E_{8}$ & $F_{4}$ & 1 & 7 & - & 14 & $\mathcal{T}(\mathbb{O}, \mathbb{A})$ & $\mathbb{O}$ \\
\hline$E_{8}$ & $E_{8}$ & 1 & 0 & - & 0 & & $\mathbb{F}$ \\
\hline
\end{tabular}

TABLE 1. Gradings of the exceptional simple Lie algebras by root systems of rank at least two.

Theorem 7.1. The fine gradings, up to equivalence, of the exceptional simple Lie algebras whose universal group has free rank at least three are the following:

- The Cartan gradings of $F_{4}, E_{6}, E_{7}$, and $E_{8}$. The universal group is $\mathbb{Z}^{r}$ with $r$ the rank of the algebra.

- The gradings of $E_{r}, r=6,7,8$ induced by gradings by the root system of type $F_{4}$. The universal groups are $\mathbb{Z}^{4} \times \mathbb{Z}_{2}^{r-5}, r=6,7,8$, and the respective types are $(72,1,0,1),(120,0,3,1)$, and $(216,0,0,8)$.

- A grading of $E_{7}$ induced by a grading by the root system of type $C_{3}$. The universal group is $\mathbb{Z}^{3} \times \mathbb{Z}_{2}^{3}$ and its type is $(102,0,1,7)$.

Proof. In Table 1 the only gradings by root systems of rank at least three are the Cartan gradings, the gradings by the root system of type $F_{4}$ of $E_{r}, r=6,7,8$, and the grading by the root system of type $C_{3}$ of $E_{7}$. In the second case, the coordinate algebra is $\mathcal{H}=\mathbb{K}, \mathbb{H}$, or $\mathbb{O}$, respectively, and the only grading on these algebras with zero component equal to $\mathbb{F} 1$ are the gradings obtained by the Cayley-Dickson doubling process (see [16] or [19]), whose universal groups are $\mathbb{Z}_{2}, \mathbb{Z}_{2}^{2}$, and $\mathbb{Z}_{2}^{3}$, respectively. The computation of the types is straightforward using the model $\mathcal{T}(\mathcal{H}, \mathbb{A})$. Finally, these gradings are fine as the zero component is the Cartan subalgebra of the subalgebra $\operatorname{Der}(\mathbb{A})$ of type $F_{4}$, and the grading induced in this subalgebra is the Cartan grading, which is fine. Hence, if any of these gradings 
could be refined, the refinement would be attached to a grading by a root system of rank at least four, which is impossible.

Finally, the coordinate algebra for the grading by the root system of type $C_{3}$ of $E_{7}$ is $\mathbb{O}$. The only grading of $\mathbb{O}$ whose zero component is $\mathbb{F} 1$ is its $\mathbb{Z}_{2}^{3}$-grading. The resulting grading by $\mathbb{Z}^{3} \times \mathbb{Z}_{2}^{3}$ of $E_{7}$ is fine and its type is easily computed using the model $\mathcal{T}\left(\mathbb{O}, H_{3}(\mathbb{H})\right)$.

We finish with the promised short proofs of the classification of fine gradings for $G_{2}$ and $F_{4}$. For $G_{2}$ this was proved independently in [12] and [6], and for $F_{4}$ in [13] (see also [11] and [19]). The arguments here are very different in nature.

Theorem 7.2. Up to equivalence, the simple Lie algebra of type $G_{2}$ is endowed with two different fine gradings: the Cartan grading by $\mathbb{Z}^{2}$, and a special grading by $\mathbb{Z}_{2}^{3}$ in which the seven nonzero homogeneous spaces are all Cartan subalgebras.

Proof. Let $\Gamma: \mathcal{L}=\bigoplus_{g \in G} \mathcal{L}_{g}$ be a fine grading of the simple Lie algebra $\mathcal{L}$ of type $G_{2}$, with $G$ its universal group. By Theorem 5.1 and Table $1, \Gamma$ is the Cartan grading, the free rank of $G$ is one, or $G$ is a finite group.

If the free rank is one, then $\mathcal{L}$ is graded by the root system $B C_{1}$ (this includes gradings by $A_{1}$ ) and hence $\mathcal{L}$ is given by the generalized Tits-Kantor-Koecher Lie algebra constructed from a structurable algebra and $\Gamma$ is obtained by combining the $\mathbb{Z}$-grading given by the rank one root system, and a grading of the coordinate algebra as in Proposition 6.1. A look at the possibilities in section 8 of [2] shows that the coordinate algebra is the structurable algebra $\mathfrak{a}=\operatorname{Mat}_{2}(\mathbb{F})$ with multiplication given by

$$
\left(\begin{array}{ll}
\alpha & \beta \\
\gamma & \delta
\end{array}\right)\left(\begin{array}{ll}
\alpha^{\prime} & \beta^{\prime} \\
\gamma^{\prime} & \delta^{\prime}
\end{array}\right)=\left(\begin{array}{cc}
\alpha \alpha^{\prime}+3 \beta \gamma^{\prime} & \alpha \beta^{\prime}+\beta \delta^{\prime}+2 \gamma \gamma^{\prime} \\
\gamma \alpha^{\prime}+\delta \gamma^{\prime}+2 \beta \beta^{\prime} & \delta \delta^{\prime}+3 \gamma \beta^{\prime}
\end{array}\right)
$$

and involution

$$
\overline{\left(\begin{array}{ll}
\alpha & \beta \\
\gamma & \delta
\end{array}\right)}=\left(\begin{array}{ll}
\delta & \beta \\
\gamma & \alpha
\end{array}\right)
$$

Consider the basis $\left\{1=\left(\begin{array}{ll}1 & 0 \\ 0 & 1\end{array}\right), e=\left(\begin{array}{ll}0 & 1 \\ 0 & 0\end{array}\right), f=\left(\begin{array}{ll}0 & 0 \\ 1 & 0\end{array}\right), s=\left(\begin{array}{cc}1 & 0 \\ 0 & -1\end{array}\right)\right\}$ of $\mathfrak{a}$, so that $\mathcal{A}=$ $\operatorname{span}\{1, e, f\}$ and $\mathcal{B}=\mathbb{F} s$. Since $s^{2}=1$ and $\mathcal{B}_{0}=0, s \in \mathfrak{a}_{g}$ with $2 g=0$. The subspace $\mathbb{F} e+\mathbb{F} f=\{x \in \mathfrak{a}: s x+x s=0\}$ is graded. For any nonzero homogeneous element $\alpha e+\beta f$, the elements

$$
\begin{aligned}
s(\alpha e+\beta f) & =-\alpha e+\beta f, \\
(\alpha e+\beta f)^{2} & =2\left(\beta^{2} e+\alpha^{2} f\right)+3 \alpha \beta 1 \quad \text { and } \\
(\alpha e+\beta f)(-\alpha e+\beta f) & =2\left(\beta^{2} e+\alpha^{2} f\right)+3 \alpha \beta s
\end{aligned}
$$

are homogeneous too, and this forces the nonzero element $\beta^{2} e+\alpha^{2} f$ to be homogeneous of degree 0 and $g$ at the same time, a contradiction.

Finally, if $G$ is finite, consider the finite quasitorus $Q$ of the algebraic group $\operatorname{Aut}(\mathcal{L})$ which is the image of the character group $\hat{G}$ (isomorphic to $G$ ). Since $\Gamma$ is 
fine, $Q$ is a maximal quasitorus. Also, since $\mathcal{L}$ is of type $G_{2}, \operatorname{Aut}(\mathcal{L})$ is a connected and simply connected semisimple algebraic group. For any $\chi \in Q, \chi$ is semisimple and $\operatorname{Aut}(\mathcal{L})$ is connected and semisimple, so its centralizer $\operatorname{Cent}_{\operatorname{Aut}(\mathcal{L})}(\chi)$ is reductive (see Theorem 2.2 in [25]), and since $\operatorname{Aut}(\mathcal{L})$ is simply connected, $\operatorname{Cent}_{\operatorname{Aut}(\mathcal{L})}(\chi)$ is connected (Theorem 2.11 in $[25]$ ). Hence the solvable radical coincides with the connected component of its center, $Z\left(\operatorname{Cent}_{\operatorname{Aut}(\mathcal{L})}(\chi)\right)^{\circ}$, and this is a torus (Lemma 19.5 in [24]). As $Z\left(\operatorname{Cent}_{\operatorname{Aut}(\mathcal{L})}(\chi)\right)^{\circ}$ is contained in any maximal quasitorus of $\operatorname{Cent}_{\operatorname{Aut}(\mathcal{L})}(\chi)$, it is contained in $Q$. As $Q$ is finite, $Z\left(\operatorname{Cent}_{\operatorname{Aut}(\mathcal{L})}(\chi)\right)^{\circ}=0$, and hence $\operatorname{Cent}_{\operatorname{Aut}(\mathcal{L})}(\chi)$ is semisimple, so that the subalgebra of $\mathcal{L}$ of elements fixed by $\chi$ is semisimple.

The automorphisms of finite order of the simple Lie algebras are well known (see Chapter 8 in [27]). They are determined, up to conjugation, by a subset of nodes of the extended Dynkin diagram and some coefficients. Those automorphisms of finite order whose subalgebra of fixed elements is semisimple, correspond to the automorphisms attached to a single node. For $G_{2}$, the extended Dynkin diagram (with coefficients) is:

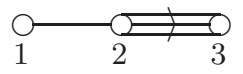

Therefore, the order of a nontrivial finite order automorphism of $\mathcal{L}$ whose subalgebra of fixed elements is semisimple is 2 or 3 . Thus $Q$ is a maximal nontoral elementary $p$-subgroup of $\operatorname{Aut}(\mathcal{L})$, with $p=2$ or 3 . According to [22], there is just one possibility, up to conjugation, where $Q$, and hence $G$, is isomorphic to $\mathbb{Z}_{2}^{3}$.

Theorem 7.3. Up to equivalence, the simple Lie algebra $\mathcal{L}$ of type $F_{4}$ is endowed with four different fine gradings, whose universal groups and types are as follows:

- the Cartan grading by $\mathbb{Z}^{4}$, of type $(48,0,0,1)$;

- a grading by $\mathbb{Z} \times \mathbb{Z}_{2}^{3}$, of type $(31,0,7)$;

- a grading by $\mathbb{Z}_{2}^{5}$ of type $(24,0,0,7)$; and

- a grading by $\mathbb{Z}_{3}^{3}$ of type $(0,26)$, such that for any $0 \neq \alpha \in \mathbb{Z}_{3}^{3}, \mathcal{L}_{\alpha} \oplus \mathcal{L}_{-\alpha}$ is a Cartan subalgebra of $\mathcal{L}$.

Proof. Let $\Gamma: \mathcal{L}=\bigoplus_{g \in G} \mathcal{L}_{g}$ be a fine grading of the simple Lie algebra $\mathcal{L}$ of type $F_{4}$, with $G$ its universal group. By Theorem 5.1 and Table 1, one of the following is true: $\Gamma$ is the Cartan grading, the free rank of $G$ is two and $\Gamma$ is associated to a grading by the root system of type $G_{2}$, the free rank of $G$ is one, or $G$ is a finite group.

If the free rank of $G$ is 2 , the the coordinate algebra (see Table 1 ) is the Jordan algebra $H_{3}(\mathbb{F})$ of symmetric $3 \times 3$ matrices. However, the results of [5] show that the zero component of any grading on $H_{3}(\mathbb{F})$ by any group has dimension at least 3 , and this contradicts Proposition 6.1.

If the free rank of $G$ is one, then $\mathcal{L}$ is graded by the root system $B C_{1}$ (this includes gradings by $A_{1}$ ) and hence $\mathcal{L}$ is given by the Tits-Kantor-Koecher Lie 
algebra constructed from a structurable algebra and $\Gamma$ is obtained by combining the $\mathbb{Z}$-grading given by the rank one root system and a grading of the coordinate algebra as in Proposition 6.1. A look at the possibilities in section 8 of [2] shows that the coordinate algebra is either the Cayley algebra $\mathbb{O}$, with its standard involution, or a structurable algebra defined on the vector space of matrices $\left(\begin{array}{cc}\alpha & a \\ b & \beta\end{array}\right)$, with $\alpha, \beta \in \mathbb{F}$ and $a, b \in H_{3}(\mathbb{F})$.

For the Cayley algebra, there is a unique grading, up to equivalence, whose zero component is $\mathbb{F} 1$, with universal group $\mathbb{Z}_{2}^{3}$, and this yields the grading by $\mathbb{Z} \times \mathbb{Z}_{2}^{3}$.

In the second case, the coordinate algebra $\mathfrak{a}=\mathcal{A} \oplus \mathcal{B}$ has dimension 14, with $\operatorname{dim} \mathcal{B}=1$. Moreover, $\mathcal{B}=\mathbb{F} s$ for an element $s$ with $s^{2}=1$, and hence $\mathcal{B}=\mathcal{B}_{g}$ for an element $0 \neq g \in \operatorname{tor}(G)$ with $2 g=0$. The Lie algebra $\mathcal{L}$ decomposes as in (3.4), and the zero component of the associated grading by the root system of type $B C_{1}$ decomposes as

$$
\mathcal{L}(0) \simeq \mathcal{A} \oplus \mathcal{B} \oplus \mathcal{D} \simeq \mathfrak{a} \oplus \mathcal{D}
$$

This is a reductive Lie algebra with one-dimensional center (corresponding to $\mathbb{F} 1$ ) and derived subalgebra simple of type $C_{3}$. (Actually $\mathcal{L}(0)$ is isomorphic to the structure Lie algebra $\operatorname{Str}(\mathfrak{a},-)$, see section 1 in [2].) On the other hand, $\mathcal{D}$ is isomorphic to the Lie algebra of derivations of $\mathfrak{a}$, which is simple of type $A_{2}$. The results in [18] show that the simple Lie algebra of type $C_{3}$ is endowed with a unique grading with trivial zero component, with universal group $\mathbb{Z}_{2}^{4}$ and type $(12,0,3)$. On the other hand, the simple Lie algebra of type $A_{2}$ has a unique grading, up to equivalence, with trivial zero component and whose universal group is 2-elementary. Its type is $(6,1)$. It occurs that $\operatorname{tor}(G)$ is 2-elementary and that at least two of the three homogeneous components of $[\mathcal{L}(0), \mathcal{L}(0)]$ of dimension 3 intersect the graded subspace $\mathfrak{a}$ in $(7.3)$ with dimension at least 2 . We conclude that there is an element $0 \neq h \in \operatorname{tor}(G)$ such that $\operatorname{dim} \mathcal{A}_{h} \geq 2$, and $h \neq g$ (recall $\left.\mathcal{B}=\mathcal{B}_{g}\right)$. Since $\mathfrak{a}$ is a simple structurable algebra, the form $\langle x, y\rangle=\operatorname{trace}\left(L_{x \bar{y}+y \bar{x}}\right)$ is nondegenerate [4]. However, $\left\langle\mathfrak{a}_{g_{1}}, \mathfrak{a}_{g_{2}}\right\rangle=0$ unless $g_{1}+g_{2}=0$. Therefore, the restriction of this form to $\mathcal{A}_{h}$ is nondegenerate. Now, for any two elements $x, y \in \mathcal{A}_{h}, x y \in \mathfrak{a}_{0}=\mathbb{F} 1$, so $x y=\alpha 1=\overline{x y}=\bar{y} \bar{x}=y x$ and $\langle x, y\rangle=\operatorname{trace}\left(L_{2 \alpha 1}\right)=$ $2 \alpha \operatorname{dim} \mathfrak{a}$. We can then find elements $x, y \in \mathcal{A}_{h}$ with $x^{2}=0=y^{2}$ and $x y=1$. Then the derivation $D_{x, y}$ in (3.5) satisfies $D_{x, y}(x) \neq 0$, so $0 \neq D_{x, y} \in \mathcal{D}_{0}$, a contradiction with $\mathcal{D}_{0}=0$.

We are left with the case in which $G$ is finite. As in the proof of Theorem 7.2, we consider the extended Dynkin diagram

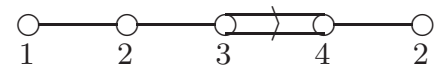

and check that either $G$ is an elementary 2-group or 3-group, or the associated quasitorus $Q(\simeq \hat{G})$ contains an automorphism $\chi$ of order 4 .

In the latter case, the subalgebra of elements fixed by $\chi$ is isomorphic to $\mathfrak{s l}(V) \oplus \mathfrak{s l}(W)$ with $\operatorname{dim} V=4 ; \operatorname{dim} W=2$ (see Chapter 8 in [27]); and the other eigenspaces of $\chi$ are, as modules for $\mathfrak{s l}(V) \oplus \mathfrak{s l}(W)$, isomorphic to $V \otimes W$, $\wedge^{2} V \otimes S^{2} W$, and $V^{*} \otimes W$, with respective eigenvalues $\sqrt{-1},-1,-\sqrt{-1}$. The action 
of any automorphism in the connected subgroup $\operatorname{Cent}_{\mathrm{Aut}(\mathcal{L})}(\chi)$ is determined by its restriction to $V \otimes W$. Now, it is not difficult to $\operatorname{check}$ that $\operatorname{Cent}_{\operatorname{Aut}(\mathcal{L})}(\chi)$ is isomorphic to $S L(V) \times S L(W) /\left\langle \pm\left(I_{V}, I_{W}\right)\right\rangle\left(I_{X}\right.$ denotes the identity map on the vector space $X)$. For $f \in S L(V)$ and $g \in S L(W)$, denote by $\psi_{f, g}$ the automorphism of $\mathcal{L}$ such that $\left.\psi_{(f, g)}\right|_{V \otimes W}=f \otimes g$. Moreover, $\Gamma$ induces gradings on $\mathfrak{s l}(V)$ and on $\mathfrak{s l}(W)$ with trivial zero components, induced by the projections

$$
\pi_{V}: S L(V) \times S L(W) /\left\{ \pm\left(I_{V}, I_{W}\right)\right\} \rightarrow P S L(V)=S L(V) /\left\langle\sqrt{-1} I_{V}\right\rangle
$$

(contained, up to isomorphism, in $\operatorname{Aut}(\mathfrak{s l}(V))$ ), and

$$
\pi_{W}: S L(V) \times S L(W) /\left\{ \pm\left(I_{V}, I_{W}\right)\right\} \rightarrow P S L(W)=S L(W) /\left\{ \pm I_{W}\right\} .
$$

There is [18], up to equivalence, only one possibility for such a grading on $\mathfrak{s l}(W)$, where $\pi_{W}(Q)=\left\langle\bar{g}_{1}, \bar{g}_{2}\right\rangle$, with $g_{1}, g_{2} \in S L(W)$ of order $2, g_{1} g_{2}=-g_{2} g_{1}$ and $\bar{g}_{i}$ denotes the class of $g_{i}$ in $P S L(W)$. With $g_{0}=I_{W}, g_{3}=g_{1} g_{2}$, and $Q_{V}^{i}=$ $\left\{f \in S L(V): \psi_{f, g_{i}} \in Q\right\}$ we have $Q=\cup_{i=0}^{3} \psi_{Q_{V}^{i}, g_{i}}$. Since $Q$ is abelian, $\psi_{f, g} \psi_{f, g^{\prime}}=$ $\psi_{f^{\prime}, g^{\prime}} \psi_{f, g}$, and it follows from $g_{1} g_{2}=-g_{2} g_{1}$ that the elements of $Q_{V}^{i}$ anticommute with the elements of $Q_{V}^{j}$ for $1 \leq i \neq j \leq 3$, and that the elements of $Q_{V}^{0}$ commute with the elements in any $Q_{V}^{i}$.

Now, there are [18], up to equivalence, only two possibilities for gradings on $\mathfrak{s l}(V)$ whose associated quasitorus is contained in $P S L(V)$ and whose zero component is trivial. In the first of these possibilities, $\pi_{V}(Q)=\left\langle\bar{f}_{1}, \bar{f}_{2}\right\rangle$ with $f_{1} f_{2}=\sqrt{-1} f_{2} f_{1}$ but since, by the preceding, any two elements of $\pi_{V}(Q)$ must either commute or anticommute, this is not possible. In the other possibility $\pi_{V}(Q)=\left\langle\bar{f}_{1}, \bar{f}_{2}, \bar{f}_{1}^{\prime}, \bar{f}_{2}^{\prime}\right\rangle$, with $f_{1}, f_{2}, f_{1}^{\prime}$, and $f_{2}^{\prime}$ order two elements of $S L(V)$ such that $f_{1} f_{2}=-f_{2} f_{1}, f_{1}^{\prime} f_{2}^{\prime}=-f_{2}^{\prime} f_{1}^{\prime}$ and $f_{i} f_{j}^{\prime}=f_{j}^{\prime} f_{i}$ for any $i, j=1,2$. We can assume, scaling the elements if necessary, that $f_{1} \in Q_{V}^{1}$ and $f_{2} \in Q_{V}^{2}$. Then, up to scalars, $f_{1}^{\prime}$ and $f_{2}^{\prime}$ must belong to $Q_{V}^{0}$, since they commute with both $f_{1}$ and $f_{2}$. This is a contradiction, since $f_{1}^{\prime}$ and $f_{2}^{\prime}$ anticommute.

We conclude that, if $G$ is finite, the maximal quasitorus cannot contain an automorphism of order 4, and hence $G$ is an elementary 2-group or 3-group, and the results in $[22]$ prove that either $G \cong \mathbb{Z}_{2}^{5}$ or $G \cong \mathbb{Z}_{3}^{3}$. The description of the gradings (with the exception of the Cartan grading) and their types appear, for instance, in [17].

\section{References}

[1] Allison, B. N.: A class of nonassociative algebras with involution containing the class of Jordan algebras. Math. Ann. 237 (1978), no. 2, 133-156.

[2] Allison, B. N.: Models of isotropic simple Lie algebras. Comm. Algebra 7 (1979), no. $17,1835-1875$.

[3] Allison, B. N., Benkart, G. And Gao, Y.: Lie algebras graded by the root systems $B C_{r}, r \geq 2$. Mem. Amer. Math. Soc. 751 (2002).

[4] Allison, B. N. And Schafer, R. D.: Trace form for structurable algebras. J. Algebra 121 (1989), no. 1, 68-80. 
[5] Bahturin, Y.A., Shestakov, I. P. and Zaicev, M.: Gradings on simple Jordan and Lie algebras. J. Algebra 283 (2005), no. 2, 849-868.

[6] Bahturin, Y. A. And Tvalavadze, M. V.: Group gradings on $G_{2}$. Comm. Algebra 37 (2009), no. 3, 885-893.

[7] Benkart, G. and Smirnov, O.: Lie algebras graded by the root system BC1. J. Lie Theory 13 (2003), no. 1, 91-132.

[8] Benkart, G. and Zelmanov, E.: Lie algebras graded by finite root systems and intersection matrix algebras. Invent. Math. 126 (1996), 1-45.

[9] Berman, S. and Moody, R. V.: Lie algebras graded by finite root systems and the intersection matrix algebras of Slodowy. Invent. Math. 108 (1992), 323-347.

[10] Bourbaki, N.: Lie groups and Lie algebras. Chapters 1-3. Translated from the French, reprint of the 1989 English translation. Elements of Mathematics (Berlin), Springer-Verlag, Berlin, 1998.

[11] Draper, C.: A non-computational approach to the gradings on $\mathfrak{f}_{4}$. Rev. Mat. Iberoam. 28 (2012), no. 1, 273-296.

[12] Draper, C. and Martín GonzÁlez, C.: Gradings on $\mathfrak{g}_{2}$. Linear Algebra Appl. 418 (2006), no. 1, 85-111.

[13] Draper, C. and Martín-GonzÁlez, C.: Gradings on the Albert algebra and on $\mathfrak{f}_{4}$. Rev. Mat. Iberoam. 25 (2009), no. 3, 841-908.

[14] Draper, C. and Viruel, A.: Fine gradings on $\mathfrak{e}_{6}$. To appear in Publ. Mat.

[15] Dynkin, E. B.: Semisimple subalgebras of semisimple Lie algebras. (Russian) Mat. Sbornik N.S. 30(72) (1952), no. 2, 349-462. Amer. Math. Soc. Transl. Ser. 26 (1957), 111-244.

[16] Elduque, A.: Gradings on octonions. J. Algebra 207 (1998), no. 1, 342-354.

[17] Elduque, A.: Gradings on symmetric composition algebras. J. Algebra 322 (2009), no. $10,3542-3579$.

[18] Elduque, A.: Fine gradings on simple classical Lie algebras. J. Algebra 324 (2010), no. $12,3532-3571$.

[19] Elduque, A. And Kochetov, M.: Gradings on the exceptional Lie algebras $F_{4}$ and $G_{2}$ revisited. Rev. Mat. Iberoam. 28 (2012), no. 3, 773-813.

[20] Elduque, A. And Kochetov, M.: Gradings on simple Lie algebras. Mathematical Surveys and Monographs 189, American Mathematical Society, Providence, RI, 2013.

[21] Elduque, A. and Okubo, S.: $S_{4}$-symmetry on the Tits construction of exceptional Lie algebras and superalgebras. Publ. Mat. 52 (2008), no. 2, 315-346.

[22] Griess, R. L., JR.: Elementary abelian p-subgroups of algebraic groups. Geom. Dedicata 39 (1991), no. 3, 253-305.

[23] Hesselink, W. H.: Special and pure gradings of Lie algebras. Math. Z. 179 (1982), no. $1,135-149$.

[24] Humphreys, J. E.: Linear algebraic groups. Graduate Texts in Mathematics 21, Springer-Verlag, New York-Heidelberg, 1975.

[25] Humphreys, J. E.: Conjugacy classes in semisimple algebraic groups. Mathematical Surveys and Monographs 43, American Mathematical Society, Providence, RI, 1995.

[26] Jacobson, N.: Lie algebras. Republication of the 1962 original. Dover Publications, New York, 1979. 
[27] KAC, V. G.: Infinite-dimensional Lie algebras. Third edition. Cambridge University Press, Cambridge, 1990.

[28] Patera, J. and Zassenhaus, H.: On Lie gradings. I. Linear Algebra Appl. 112 (1989), 87-159.

[29] Tits, J.: Algèbres alternatives, algèbres de Jordan et algèbres de Lie exceptionnelles. I. Construction. Nederl. Akad. Wetensch. Proc. Ser. A 69 =Indag. Math. $\mathbf{2 8}$ (1966), 223-237.

Received March 4, 2013.

Alberto Elduque: Departamento de Matemáticas e Instituto Universitario de Matemáticas y Aplicaciones, Universidad de Zaragoza, 50009 Zaragoza, Spain.

E-mail: elduque@unizar.es

Supported by the Spanish Ministerio de Economía y Competitividad and FEDER (MTM2010-18370-C04-02) and by the Diputación General de Aragón-Fondo Social Europeo (Grupo de Investigación de Álgebra). 